J Control Release. 2014 September 28; 0: 210-218. doi:10.1016/j.jconrel.2014.04.014.

\title{
GELATIN CARRIERS FOR DRUG AND CELL DELIVERY IN TISSUE ENGINEERING
}

\author{
Marco Santoro ${ }^{a}, \S$, Alexander M. Tatara ${ }^{b,}$, and Antonios G. Mikos ${ }^{a, b},{ }^{\star}$ \\ aDepartment of Chemical and Biomolecular Engineering, Rice University, Houston, TX 77005 \\ ${ }^{b}$ Department of Bioengineering, Rice University, Houston, TX 77030
}

\begin{abstract}
The ability of gelatin to form complexes with different drugs has been investigated for controlled release applications. Gelatin parameters, such as crosslinking density and isoelectric point, have been tuned in order to optimize gelatin degradation and drug delivery kinetics. In recent years, focus has shifted away from the use of gelatin in isolation towards the modification of gelatin with functional groups and the fabrication of material composites with embedded gelatin carriers. In this review, we highlight some of the latest work being performed in these areas and comment on trends in the field. Specifically, we discuss gelatin modifications for immune system evasion, drug stabilization, and targeted delivery, as well as gelatin composite systems based on ceramics, naturally-occurring polymers, and synthetic polymers.
\end{abstract}

\section{Keywords}

Gelatin; drug delivery; cell delivery; controlled release; composite material

\section{Introduction}

Among other challenges, biomedical research has been recently focused on the design of new drug delivery systems with more tunable and predictable release kinetics. Furthermore, past biotechnological advances in the production of peptides, proteins, and nucleic acids did not reflect in an equivalent development of carriers for the release of these particular classes of bioactive substances. Naturally-derived polymers, such as collagen, allow for fabrication of drug delivery systems under mild formulation conditions. However, drug delivery carriers based on naturally-derived polymers are typically hydrogel systems that rely essentially on diffusion, with low extent of versatility and reduced potential for long-term release [1].

(C) 2014 Elsevier B.V. All rights reserved.

*To whom correspondence may be addressed: Antonios G. Mikos, PhD, Department of Bioengineering, MS-142, BioScience Research Collaborative, Rice University, 6500 Main Street, Houston, TX 77030, mikos@ rice.edu; Tel: (713) 348-5355, Fax: (713) 348-4244.

$\S$ These authors contributed equally to this work

Publisher's Disclaimer: This is a PDF file of an unedited manuscript that has been accepted for publication. As a service to our customers we are providing this early version of the manuscript. The manuscript will undergo copyediting, typesetting, and review of the resulting proof before it is published in its final citable form. Please note that during the production process errors may be discovered which could affect the content, and all legal disclaimers that apply to the journal pertain. 
A substantial breakthrough in the classical design of diffusion-controlled systems comes from the use of polyion complexation-based systems, in which the drug (typically a macromolecule) electrostatically interacts with the carrier to form a polyion complex. In light of the numerous ionic interactions per each drug and carrier, it is statistically unlikely that all interactions between drug and carrier dissociate simultaneously. Hence, the resulting interactions are remarkably stable and the secondary bonds in the polyion complex do not dissociate as easily as the bonds between electrolytes with low molecular weight [2]. Several systems have been proposed as effective complexes, from cationic polymers for DNA delivery [3, 4], to natural polymers such as chitosan [5] and poly(L-lysine) [6].

Amongst the potential polyion complexation-based carriers, gelatin stands out strongly due to several key advantages. Gelatin, a natural protein derived from the hydrolysis of collagen, is highly biocompatible and biodegradable in a physiological environment [7]. Although mainly derived from animals, the digestive process confers gelatin very low antigenicity, with the formation of harmless metabolic products upon degradation $[8,9]$. In order to prevent possible infectious disease transmission, genetic engineering approaches recently emerged for the production of human recombinant gelatin [10-12]. The presence of aminoacidic sequences such as Arg-Gly-Asp (RGD) in the structure improves the final biological performance of gelatin over synthetic polymers that lack these cell-recognition motifs [13]. Finally, in no small part due to its inexpensiveness and high availability, gelatin is already widely used in pharmaceutical and medical applications and has been recognized as a GRAS (Generally Regarded As Safe) material by the United States Food and Drug Administration [9].

As a drug delivery carrier, gelatin has proven to be versatile due to its intrinsic features that enable the loading of charged biomolecules. Indeed, by selecting either alkaline or acidic treatment, the gelatin isoelectric point (IEP) can be tailored to maximize drug loading efficiency depending on the electrostatic properties of the desired drug molecule [1]. In addition, release kinetics from gelatin depends on carrier degradation, which can be tuned by either varying gelatin molecular weight or the extent of material crosslinking [2]. Accordingly, drug loading and release kinetics can be independently optimized, making gelatin an exceptionally adaptable drug delivery carrier as witnessed by the broad range of applications in which it has been used: tissue engineering [14-16], cancer therapy [17-19], and therapeutic angiogenesis [20-22].

Initial investigations focused mainly on the effect of gelatin physico-chemical properties on drug delivery kinetics, relying on the ability of gelatin to preserve the bioactivity of therapeutic agents [2, 23]. Nevertheless, the last decade witnessed the development of more advanced gelatin-based controlled release systems, with the investigation of a wider variety of bioactive agents and the synergistic use of gelatin with several other materials [9, 24]. This review will therefore focus on current advances in the design of gelatin systems for drug and cell delivery in regenerative medicine. While initial work in the field of gelatin drug delivery focused on gelatin in different geometries and IEPs [2], the newer developments revolve around gelatin modification and inclusion of gelatin in composite systems. The versatility of gelatin structure has evoked great interest, and gelatin chemical 
modification and composite system development have emerged recently as strategies to modulate drug release kinetics and to design targeted drug delivery vehicles.

\section{Modification of Gelatin}

In light of its tremendous potential for controlled release, gelatin has been extensively investigated as a delivery vehicle for many classes of drugs, including but not limited to antibacterial agents [25, 26], anti-inflammatory drugs [27, 28], and many different antineoplastic compounds $[17,19,29,30]$. Regardless of the specific application, gelatin carriers demonstrate reduced side effects when compared to the administration of the free compound, with improved pharmacokinetic profile and drug efficacy [9]. Recent reports in the literature have focused on expanding the use of gelatin to more sensitive drugs, such as nucleic acids and hydrophobic compounds [31, 32]. As such, chemical motifs have been introduced to the flexible aminoacidic structure of gelatin in order to ensure high encapsulation efficiency and the desired release of these drugs [33]. Modification of gelatin follows three strategies: (i) the synthesis of stealth carriers to increase drug bioavailability [17], (ii) the enhancement of drug encapsulation efficiency [34], and (iii) the conjugation of gelatin with dedicated biomolecules for targeted drug delivery [35] (Table 1).

\subsection{Stealth Delivery}

It is widely recognized that drug delivery carriers (especially at the micro- to nano-scale) are rapidly identified and phagocytized by the reticuloendothelial system, depending on their physicochemical properties such as particle size, hydrophobicity and zeta potential [17]. The modification of gelatin with poly(ethylene glycol) (PEG), termed PEGylation, is currently the leading strategy for the fabrication of stealth drug delivery systems, with slower clearance from the body and prolonged circulatory time [43]. PEG hydrophilicity precludes adsorption of proteins on the carrier due to its steric repulsion effect, thus preventing opsonization and reducing immunogenicity $[17,44]$. The addition of PEG to gelatin is easily facilitated as the primary amine groups on the gelatin amino acid backbone can react with the addition of PEG-epoxide or PEG-dialdehyde, allowing for site-specific modification by PEG [45, 46]. Physicochemical analysis of PEGylated gelatin demonstrated increased time to reach maximum swelling ratio and time to failure by hydrolysis, with no significant impact on maximum swelling weight ratio or swelling weight ratio at failure, compared to unmodified gelatin [46].

Lee et al. investigated PEGylation of gelatin by comparing the behavior of PEG-modified and unmodified gelatin nanoparticles for the delivery of doxorubicin, a drug clinically used for oncological applications [36]. The PEG-gelatin carrier was found to have significantly decreased cytotoxicity than doxorubicin intravenous administration according to hematological and histological findings in a mouse model for pulmonary metastasis [36]. Both nanoparticle systems exhibit significant inhibition of tumor growth and reduced pulmonary metastasis in vivo with no statistical difference between modified and unmodified gelatin [36]. An analogous carrier has been further developed by Kommareddy and Amiji, based on PEGylated-thiolated gelatin for DNA delivery [37]. Thiolation leads to the formation of disulfide bonds within gelatin, improving stabilization of the nanoparticles in the bloodstream [37]. Moreover, the disulfide bond can be easily broken by glutathione, 
whose concentration is usually 1000-fold higher inside cells. As a result, thiolated-gelatin allows DNA release triggered by intracellular encapsulation. Thiolated-gelatin also increases material stability (compared with gelatin) due to from additional crosslinking. Lastly, thiolated-gelatin in combination with PEG demonstrated reduced cytotoxicity [37].

Evasion of reticuloendothelial uptake is particularly crucial for tumor applications, where the longevity of the drug delivery carrier in the circulatory system allows for its accumulation in the leaky vasculature of tumor tissues, according to the enhancedpermeability effect (EPR) [47]. Along this direction, Madan et al. characterized PEGmodified gelatin microassemblies, both in vitro and in vivo, for the delivery of noscapine to human non-small cell lung carcinoma [17]. Similarly to the study of Lee et al. [36], the gelatin carrier proved to be less cytotoxic and more efficacious than free drug intravenous administration [17]. Moreover, PEGylation enhances the selectivity of the gelatin nanoparticles toward the target organ (lungs) when compared to the unmodified gelatin carrier [17]. Pharmacokinetic analyses correlate these results to an increased plasma half-life of noscapine in the PEG-modified gelatin carrier, thus resulting in a higher possibility for the carriers to be subjected to the EPR effect [17].

While current research in the oncological field is centered on gelatin modification for targeted drug delivery (discussed later in section 2.3) rather than on EPR-mediated tumor targeting [48], sterically stable gelatin nanocarriers are already translating to the clinical setting. In a recent study, Narayanan et al. loaded PEGylated gelatin nanoparticles with ibuprofen sodium [28]. This drug delivery vehicle allowed for sustained delivery of the antiinflammatory agent and minimization of side-effects such as gastric irritation and ulceration [28]. Moreover, the enhanced therapeutic benefit avoided the need for multiple injections, resulting in improved patient compliance [28]. The modification of gelatin by PEGylation enhances many of the strengths of gelatin as a drug delivery vehicle and will continue to be investigated and translated into the clinic.

\subsection{Enhanced Drug Stabilization and Loading Efficiency}

Another complementary approach aimed at improving in vivo drug bioavailability involves the modification of gelatin to increase drug loading efficiency [1, 34, 38]. The work of Kimura and Tabata demonstrated how the structure of gelatin can be tuned according to the selected drug, resulting in different electric charge density and hydrophobicity of the carrier [20]. In a recent study, six gelatin derivatives with different IEPs were compared for the delivery of stromal-cell-derived factor-1 (SDF1), a chemokine involved in angiogenesis [20]. Specifically, negatively-charged succinylated gelatin was found to be the most suitable for the delivery of SDF1, a protein positively charged at physiological $\mathrm{pH}$. Tests in mice revealed that drug release was controlled by gelatin hydrogel degradation rather than simple diffusion, with increased angiogenic activity (as measured by amount of newly-formed capillaries in implanted skinfold chambers) in the experimental groups using modified gelatin. This investigation provides a good example of gelatin structure optimization for drug delivery, with the simultaneous analysis of the different gelatin derivatives on drug activity and in vivo efficacy [20]. A similar rationale has been applied to other compounds, such as enzymes [49], and growth factors [20]. 
In the case of plasmids and DNA, a standard approach involves the use of a positively charged carrier able to stably condense with the negatively charged nucleic acid [31, 34]. Cationic gelatin, where the introduction of amine residues on the carboxyl groups increases the polymer positive charge, has been successfully employed for delivery of small interfering RNA (siRNA) [38, 50], as well as intracellular DNA [51, 52]. Several agents have been used to "cationize" gelatin, such as ethylenediamine [38, 49], polyethylenimine $[50,53]$, and spermine $[9,54,55]$.

Obata et al. used cationized gelatin microspheres to deliver siRNA to prevent the progression of peritoneal fibrosis in mice [34]. A single submesothelial injection of gelatin microparticles provided continuous release of siRNA up to three weeks, dependent on the tuned gelatin degradation rate. Immunohistochemical analyses confirmed the prevention of peritoneal fibrosis as demonstrated by the suppression of type III collagen, the limited infiltration of macrophages, and slower myofibroblast proliferation [34]. This carrier was further investigated to determine the effect of gelatin crosslinking density on siRNA intracellular delivery [38]. Changes in crosslinker concentration affected degradation kinetics and drug release but did not alter siRNA entrapment efficiency. Direct quantification of siRNA internalization and gene expression in colon cells indicated that the released siRNA maintained its biological activity, with gene silencing efficacy directly proportional to crosslinker concentration [38].

Uesugi et al. investigated dual modification of gelatin by both general strategies outlined above, as cationized gelatin was grafted with PEG chains for the delivery of tissue-type plasminogen activator (tPA) [49]. tPA is a thrombolytic agent utilized frequently in the clinic, but an excessive dosage leads to bleeding complications. However, the complexation of tPA with cationized/PEGylated gelatin can mitigate the risk of tPA-induced bleeding. This study demonstrated a strong complexation of the enzyme with the carrier, resulting in a longer bioavailability in vivo but also a remarkable suppression of tPA activity [49]. Nevertheless, tPA activity was fully restored in a rabbit model by using ultrasound irradiation, as shown by restored thrombolytic activity and full recanalization [49]. This is because the electrostatic interactions in the complex are stable enough to suppress tPA activity, but not so strong to prevent dissociation by ultrasound. Moreover, the radiation intensity did not compromise the biological activity of the released tPA [49].

The synergistic use of polyion complexation and ultrasound-triggered drug delivery, already explored in literature [56], is therefore another possibility to precisely control drug delivery kinetics while simultaneously avoiding adverse effects arising from drug burst release [49]. The combination of gelatin and poly(ethylenimine) (PEI) is an alternative strategy to prepare DNA delivery systems. PEI has high transfection efficiency but its intrinsic cytotoxicity is of great concern [3]. The use of PEI-modified gelatin systems bypasses this issue, improving simultaneously plasmid entrapment efficiency of pure gelatin [9]. Mimi et al. implemented this strategy and reported a novel core-shell nanocarrier for siRNA delivery [50]. The presence of a gelatin core posed steric limitations to the PEI molecules in the outer shell, thus limiting PEI interaction with cell membranes [50]. As a result, PEI-gelatin nanoparticles exhibited a cytotoxicity four times lower than PEI control nanoparticles, without any detrimental effect on siRNA uptake by the cells. Interestingly, gene silencing 
efficacy of the PEI-modified gelatin was considerably higher than that of a commercially available transfection agent [50].

While combining PEI or PEG with gelatin is the most prevalent modification design, other polymers have been successfully conjugated to gelatin with the ultimate goal of synthesizing amphiphilic derivatives $[32,39,57,58]$. In the last decade, many water-insoluble drugs have been developed through pharmaceutical research. In light of their physicochemical characteristics, hydrophobic polymers have been commonly used for the controlled delivery of this class of compounds. Nonetheless, hydrophobic carriers are affected by strong protein adsorption, which alters drug release kinetics and can result in stronger adverse inflammatory reactions in vivo [32]. Gelatin-based block copolymers can overcome these issues, as the inner core provides a hydrophobic domain to accommodate the drug, while the outer gelatin layer accounts for enhanced biocompatibility and faster biodegradability [32, 57].

Tanigo et al. reported one of the first attempts to deliver simvastatin, a hydrophobic hypolipidemic drug from hydrogels [32]. Briefly, gelatin was modified with lactic acid (LA) oligomers of different molecular weight in order to form micelles loaded with simvastatin. The nanocarriers were then dispersed in a crosslinked gelatin matrix, and biological activity of the overall drug delivery system was assessed. The authors emphasized how drug release was governed solely by the degradation of gelatin hydrogel, as the generation of watersoluble gelatin fragments enabled the release of drug-loaded micelles [32]. In vitro analysis of bone morphogenetic protein-2 (BMP-2) expression confirmed that the released simvastatin maintained its biological activity, as well as a prolonged release due to a higher stability of simvastatin-loaded micelle in the culture medium [32]. Copolymers of gelatin and LA have been utilized by the same group to enhance bone regeneration via dual delivery [16]. Amphiphilic hydrogels loaded with SEW2871 (a macrophage chemoattractant) and platelet-rich plasma acted synergistically in vivo, leading to improved bone regeneration compared to single agent administration [16]. This study demonstrated the effects of macrophage recruitment on bone inflammation and osteoclastic activity, generating deeper insight on the overall bone healing process [16].

In addition to these studies, the interest for amphiphilic gelatin in drug delivery has grown exponentially, with a multitude of approaches present in literature. Li et al. designed nanoparticles to entrap the hydrophobic anticancer agent camptothecin by grafting hexanoyl anhydrides to the amino groups of gelatin [39]. The degree of hexanoyl substitution strongly influenced camptothecin stability and release profile, thus emphasizing the potential tunability of drug delivery kinetics for hexanoyl-gelatin carriers [39].

Recently, another group explored the possibility to prepare self-assembled nanoparticles made of gelatin and oleic acid to improve the solubility of poorly water soluble drugs [58]. Similarly to the previously described study, a simple modification protocol was developed where oleic acid conjugation is mediated by the presence of monoethanolamine. The resulting system exhibited self-assembling properties upon dispersion in an aqueous medium [58]. Moreover, gelatin/oleic acid nanoparticles were used as a carrier to investigate the solubility and degradation rate for three model drugs and demonstrated great potential for 
both gastric and intestinal drug delivery [58]. Future strategies will likely expand current knowledge on the production of gelatin-based micelles, tailoring gelatin modification in relation to the specific drug loaded.

\subsection{Targeted Drug Delivery}

The development of targeted drug delivery systems is a central challenge in the scientific community, especially in oncology, in light of the tremendous clinical implications [59]. The possibility to selectively deliver therapeutic agents to a specific tissue allows for higher therapeutic efficacy as well as reduced side-effects and systemic toxicity. Gelatin flexibility has therefore been leveraged to introduce specific motifs for highly selective cell targeting to several organs [9].

Magadala and Amiji presented one of the first attempts to introduce epidermal growth factor receptor (EGFR)-recognition sequence onto the gelatin backbone, in order to study gene delivery in pancreatic cancer cells [40]. EGFR is highly expressed on different cancer cells and the expression level can directly correlate with tumor stage [60]. It is therefore a key target in several drug delivery approaches [35, 40, 41]. In this study, gelatin nanoparticles were grafted with PEG oligomers and further processed to introduce sequences which covalently link to EGFR-targeting peptide. EGFR-modified gelatin shows minimal cell cytotoxicity with considerably enhanced transgene expression efficiencies compared to control gelatin or PEG-modified gelatin nanoparticles [40].

Two different investigations by Tseng et al. corroborate the potential in EGFR targeting, specifically applied for the treatment of lung cancer [35, 41]. In this case, gelatin was modified with avidin moieties and then exposed to biotinylated epidermal growth factor (EGFb). The final binding between biotin and avidin groups resulted in synthesis of EGFbiotinylated gelatin (EGFb-gelatin) [41]. Aerosol delivery in a mouse model exhibited strong selectivity of EGFb-modified gelatin for lung cancer cells, with maintained bioactivity of EGF ligand following aerosolization and internalization in alveolar walls. When compared to PEGylated gelatin carriers for drug targeting based on the EPR effect, EGFb-modified carriers demonstrated not only lower accumulation in liver and kidney, but also faster accumulation in the target organ (lung) [41]. In light of these promising results, EGFb-gelatin nanoparticles were employed as carriers for the chemotherapeutic drug cisplatin [35]. Subcutaneous administration in a mouse model revealed robust antitumor activity of the cisplatin-loaded EGFb-gelatin, with higher efficiency than free cisplatin administration or unmodified cisplatin-loaded gelatin nanoparticles [35]. This system was also evaluated after aerosol delivery in a mouse lung cancer model. EGFb-gelatin carriers remained still highly selective for lungs, with lower nephrotoxicity than the actual subcutaneous administration of the same drug delivery vehicle [35].

Tian et al. recently proposed another remarkable example of gelatin-based targeted drug delivery [42]. Drug delivery through the blood-brain barrier (BBB) is a major concern for the pharmaceutical community and current carrier options are limited, due to low permeability. This study utilized all three strategies described in this section, as gelatin was conjugated with both PEG and Tat peptide, a protein regularly utilized to enhance brain drug delivery via transcytosis [61]. In vivo organ distribution indicated that PEG-Tat-gelatin 
nanoparticles preferentially accumulated in the brain rather than liver and spleen when compared to simple PEG-gelatin carriers. Moreover, ex vivo imaging displayed a strong localization of PEG-Tat-gelatin nanoparticles throughout the brain, while PEG-gelatin carriers exhibited much weaker signal [42]. These results prove how the strategy of active targeting can be used with gelatin-based delivery systems to facilitate carriers not only evading reticuloendothelial clearance, but also crossing the $\mathrm{BBB}$ to reach the central nervous system [42].

\section{Gelatin-based composites}

Another important research direction involves the study of gelatin within composite material systems for the controlled delivery of therapeutics and cells. Composite systems synergistically combine two or more materials in order to produce a new system with novel properties unique to either material alone, such as extended release kinetics or increased mechanical properties. As a mark of its versatility, gelatin has been demonstrated to be a useful component in composites featuring materials ranging from ceramics to natural and synthetic polymers (Table 2).

\subsection{Ceramics}

While most of the tissues in the body are composed of proteins, the skeleton is a composite system itself, consisting of a protein-based polymer (collagen) coated in a ceramic (hydroxyapatite). Accordingly, there have been many attempts to create similar composite systems with gelatin and ceramics in order to deliver drugs and otherwise promote bone regeneration. Two of the most challenging clinical complications resulting from bone defects are wound infection and non-union [66]. As a result, the two most explored types of drugs delivered by ceramic/gelatin composite systems are antibiotics and growth factors.

3.1.1. Hydroxyapatite-Hydroxyapatite, $\mathrm{Ca}_{10}\left(\mathrm{PO}_{4}\right)_{6}(\mathrm{OH})_{2}(\mathrm{HA})$ is a naturally-occurring mineral which comprises the inorganic component of bone matrix. As an example of a simple two phase composite, porous HA can be coated with gelatin in order to increase construct mechanical properties [67]. This increase was show be proportional to the concentration of gelatin, leading the authors to hypothesize that gelatin increases HA toughness by bridging material cracks [67]. More advanced fabrication techniques have been employed, where HA nanoparticles have been precipitated within gelatin networks to form scaffolds capable of drug delivery. The resulting nano-composite constructs had high porosity ( $\sim 90 \%$ ), ordered structure, and most importantly the capability to control antibiotic drug delivery (tetracycline) via both gelatin crosslinking density and amount of HA incorporation [68].

Using a similar HA precipitation-based strategy, porous HA-gelatin microparticles were fabricated with fairly uniform size distribution in a one pot reaction using vegetable oil rather than harsh organic solvents [69]. Alternatively, HA-gelatin microparticles have been synthesized by addition of granulated coralline HA to a solution of gelatin, and then adding the mixture dropwise to a polymethylmethacrylate (PMMA) dispersion and crosslinking via glutaraldehyde [70]. After washing and drying, these microparticles were loaded by immersion into solution containing water-soluble drug (in this case, the antibiotic 
gentamicin). This second method of HA-gelatin composite microparticle synthesis results in more variable microparticle size, with diameters ranging from 1-100 $\mu$ m (mean size as 16 $\mu \mathrm{m})$ but has demonstrated drug release for up to three days. While these microparticles may have desirable properties for controlled release of drugs, larger particle sizes may be more suitable for delivery of cells. HA-gelatin microparticle size was tuned via stirring speed at the time of synthesis. After synthesis, the microparticles were seeded with human osteoblast-like Saos-2 cells. Compared with HA microparticles without gelatin, cells seeded on composite microparticles experienced greater proliferation, demonstrating that this gelatin-HA system could potentially be used as an injectable carrier for cell delivery [71].

3.1.2. Calcium Phosphate-Calcium phosphate cements (CPCs) are mixtures of calcium orthophosphates that can be injected to fill craniofacial and orthopedic defects. CPCs have also been used as drug delivery vehicles by incorporating drug in the liquid or solid phase of the cement before setting [72]. While CPCs have had great success in the clinic due to inherent osteoconductivity and ease of material delivery by injection, lack of biodegradation inhibits replacement by native tissue and limits drug release to diffusion [62, 72]. Habraken et al. incorporated degradable gelatin microparticles into CPCs in order to generate macroporosity following microparticle degradation to encourage tissue integration [62]. Subsequently, the embedded gelatin microparticles within this composite system were used not only as porogens but also as active drug delivery vehicles.

Transforming growth factor-beta (TGF- $\beta$ ), basic fibroblast growth factor (bFGF), and BMP-2 were loaded into microparticles made of acid-treated gelatin or alkali-treated gelatin, which were subsequently loaded into CPC and evaluated for drug release over 45 days in vitro [63]. As a control, growth factors were encapsulated and absorbed by CPC without microparticles. By the end of the investigation, all gelatin microparticle groups had only released a third or less of their total growth factor load, with sustained drug release (drug absorbed onto CPC surface showed over $60 \%$ release and drug encapsulated in CPC showed release similar to microparticles). In a goat vertebrae defect model, CPC was loaded with either BMP-2 alone or gelatin microparticles loaded with BMP-2 [73]. Gelatin microparticles showed more rapid release (45\% release over 28 days) and vertebrae treated with gelatin-CPC composites had greater pushout strength at 45 and 140 days after surgery and a greater mineralization rate at 45 days after surgery compared to CPC loaded with BMP-2 alone [73]. While these data appear promising, it is worth noting that unloaded CPCgelatin composite microparticles induced greater inflammation than blank PLGA microparticles embedded within CPC, and resulted in less material replacement by newly formed bone in a rabbit femoral defect [74]. This inflammation may have been due to immunogenic response to porcine gelatin in a rabbit animal model. Deeper in vivo characterizations are required in order to elucidate gelatin potential as a porogen and drug release carrier into CPCs.

\subsection{Naturally-derived Polymers}

Naturally-occurring polymers such as collagen are the building blocks of the extracellular matrix and are therefore attractive materials to use for drug delivery and tissue engineering [75]. These materials include polymers synthesized by human cells (such as collagen and 
hyaluronan) as well as naturally-derived polymers synthesized by other organisms (such as chitin and silk) [76, 77]. Composites of gelatin and other naturally-occurring polymers offer good biocompatibility and enable biomimetic strategies for drug delivery.

3.2.1. Hyaluronan-Hyaluronan is a ubiquitous polysaccharide with a high density of negative charge (allowing for water retention) and the ability to act as a binding site for molecules, making it an attractive biomaterial for drug delivery and tissue engineering [7880]. However, the hydrophilicity of hyaluronan can inhibit protein absorption and prevent cell attachment. Therefore, gelatin-hyaluronan composites have been explored in order to take advantage of the strengths of both these materials. One technique for composite synthesis is incorporation of thiol functional groups onto both the gelatin and hyaluronan to allow for crosslinking [81]. Incorporating gelatin into hyaluronan scaffolds increase seeded cell proliferation in a dose-dependent fashion [81].

In order to facilitate the controlled delivery of growth factors from these gelatin/hyaluronan composite hydrogels, Peattie et al. incorporated thiolated heparin into the matrix [82]. These scaffolds were successful in delivering a wide variety of growth factors over at least two weeks, and the incorporation of gelatin increased the drug delivery rate [82]. These gelatinhyaluronan-heparin composites also demonstrated efficacy in a mouse model, delivering multiple growth factors concurrently to stimulate blood vessel formation near the murine ear [83]. In addition to thiol modification, gelatin and hyaluronan have been blended together by electrospinning to create biodegradable sheets with tunable surface tensions that may have future use as drug delivery vehicles [84].

3.2.2. Chitin and Chitosan-Chitin is a naturally-derived polysaccharide synthesized by a variety of organisms, including crustaceans and some fungi. For use as a drug delivery vehicle and cell scaffold, it is often modified by alkaline deacetylation to form chitosan [85]. Like hyaluronan, chitosan has a high charge density and good biocompatibility. Gelatin and chitosan form polyionic complexes and therefore composite construct drug release kinetics and degradation can be modified by amount of gelatin incorporation [86]. As release kinetics of these types of constructs are $\mathrm{pH}$-dependent, with decreased $\mathrm{pH}$ resulting in faster release, gelatin-chitosan sponges have been investigated for use as release vehicles for wound treatment [64]. HA was introduced to these sponges, resulting in a material conducive for osteoblast growth $[87,88]$. In addition to scaffolds and sponges, gelatin/ chitosan microparticles have been successfully designed, with sustained delivery of bFGF for over 2 weeks in vitro [89].

3.2.3. Silk-Unlike the polysaccharides hyaluronan and chitin, silk is a protein-based polymer, produced by insects and spiders. In recent years, silks and silk-derivatives have been studied in tissue engineering as lightweight yet tough biomaterials [90]. For this reason, silk has been used to reinforce gelatin scaffolds, resulting in greater tensile and bending strength [91]. Mandal et al. synthesized gelatin-silk composites that can be loaded with water soluble drug and fabricated in stackable layers [92]. These constructs were capable of releasing model drug for at least 25 days with release kinetics and degradation tunable by ratio of gelatin to silk [92]. 
Gelatin-silk hydrogel composites were also synthesized into hydrogels which gel upon contact with aqueous methanol [93]. This was due to the solution inducing transformation of the silk from random-coil to $\beta$-sheet conformation, causing physical crosslinking of the hydrogel. These hydrogels were thermally-responsive and when temperature was increased from $20^{\circ} \mathrm{C}$ to $37^{\circ} \mathrm{C}$, construct swelling greatly increased and the hydrogels experienced greater mass loss due to gelatin release [94]. The amount of mass loss was proportional to the amount of gelatin in the construct and this tunable thermal activation may prove useful in designing drug delivery vehicles which respond specifically to physiologic temperatures. Gelatin-silk composites combine the strength of silk with the favorable biodegradation profile and drug-loading capability of gelatin to create versatile constructs.

\subsection{Synthetic Polymers}

One of the key strengths of synthetic polymers in drug delivery is the ability to tune and modify material chemistry [95]. However, without specific design considerations, most synthetic polymers inherently lack cell-recognition sequences and binding sites, eliciting inflammatory response and foreign body reaction $[95,96]$. Therefore, the synergy of gelatin, a protein-based material with many cell-recognition sites, combined with synthetic polymers can result in a highly customizable platform for drug delivery.

3.3.1. Poly(lactic-co-glycolic acid) (PLGA)_PLGA is one of the most prevalent synthetic polymers applied in the field of drug delivery and tissue engineering. It is a component of several FDA-approved delivery devices, such as in antibiotic-releasing microspheres for periodontal application [97]. The primary mechanism of drug release from PLGA is diffusion mediated by matrix degradation, which can be tuned by factors such as molecular weight, ratio of lactic to glycolic acid, and chain end group [98]. However, as a synthetic polymer, PLGA does not inherently possess cell-adhesion motifs. Therefore, gelatin-PLGA composite microparticles were synthesized by emulsifying gelatin in a PLGA/dichloromethane solution and following standard water/oil/water emulsion microparticle synthesis techniques [65]. RGDS, a peptide ligand sequence, was attached to the gelatin portion of the microparticles via carbodiimide. Chondrocytes cultured on gelatinPLGA microparticles had higher viability, greater proliferation, and larger deposition of glycosaminoglycans compared to PLGA microparticles alone [65]. Incorporation of RGDS motifs further statistically increased cell proliferation and viability. This promising composite system is also injectable and could potentially be utilized for the controlled delivery of chondrocytes to articular defects [65].

Another way in which PLGA and gelatin have been combined for controlled delivery is through electrospinning techniques. Drug delivery through encapsulation within electrospun fibers is appealing due to the high surface-to-volume ratio of electrospun constructs [99]. By electrospinning a composite of gelatin and PLGA dissolved in 2,2,2-tri uoroethanol, drug release of encapsulated fenbufen was able to be tuned by fiber alignment and gelatin crosslinking time via glutaraldehyde vapor [100]. Gelatin-PLGA fibers also release drug in a $\mathrm{pH}$-sensitive manner and show promise as a tunable drug carrier. The unloaded fibers have also been characterized for mechanical properties and ability to support pre-osteoblasts in vitro, where composite fibers featured greater cell adhesion and proliferation than PLGA 
alone [101]. In general, incorporation of gelatin into PLGA constructs allows for further mechanisms to tune release kinetics as well as increases cell biocompatibility.

3.3.2. Oligo(poly(ethylene glycol) fumarate) (OPF)—Crosslinked OPF is a PEGbased hydrogel with cleavable fumarate groups, allowing for biodegradation, unlike PEG [102]. The properties of OPF can be controlled by varying molecular weight, crosslinking density, and type of crosslinker molecules [103]. OPF has been demonstrated to support controlled drug release, cell encapsulation [104], and tissue regeneration [105]. However, greater success within the field of drug delivery has been accomplished using OPF hydrogels with the inclusion of embedded gelatin microparticles. These composite systems have been utilized for the controlled delivery of molecules such as DNA [106], minerals such as HA [107], and numerous growth factors [15, 108], in in vitro and in vivo models. While drug release from OPF alone is mainly regulated by molecular diffusion, introduction of drug-loaded gelatin microparticles allows for formation of polyionic complexes and significantly reduces burst release from gelatin microparticles [109]. By encapsulating gelatin microparticles with different loaded drugs in the same composite, multiple therapeutics can be delivered concurrently [108].

To this effect, gelatin microparticles loaded with either insulin-like growth factor 1 (IGF-1), TGF- $\beta 3$, or both were encapsulated in either the cartilage layer of a bilayered OPF construct for treatment of a rabbit osteochondral defect. In other bilayered constructs, the growth factors were loaded directly into the OPF gel phase [15]. Constructs were able to release growth factor in vitro for at least 25 days and drug released from gelatin encapsulated within OPF retained greater bioactivity compared to drug released from OPF alone. In the animal model, greater frequency of hyaline-like cartilage was observed when TGF- $\beta 3$ was delivered in the presence of IGF-1 [15]. Research in the area of gelatin-OPF composite constructs for controlled drug delivery is ongoing and has great promise for the generation of complex tissues, such as the osteochondral junction.

3.3.3. Poly(propylene fumarate) (PPF)—Similar to OPF, PPF is a synthetic polymer that contains fumarate and biodegradable ester bonds. However, PPF has greater hydrophobicity than OPF and has greater stiffness and strength [110, 111]. PPF has demonstrated to be photocrosslinkable [112], biocompatible in soft and hard tissues [113], osteoconductive [114], and effective in regenerating bone in a variety of animal models $[111,115,116]$. The positive attributes of PPF as a substrate, combined with the controlled delivery of gelatin microparticles, have been synergized by encapsulating gelatin microparticles within porous PPF [117]. BMP-2 and VEGF were delivered both simultaneously and individually in a critically-sized rat cranial defect to study the effects of dual delivery on bone regeneration. BMP-2 alone and dual delivery groups elicited significant bone and blood vessel formation (BMP-2 had a dose-dependent effect) compared to VEGF alone and blank controls $[117,118]$. Interestingly, in this animal model, dual delivery of growth factors was not advantageous over BMP-2 alone at later time points.

An alternative approach to delivering BMP-2 and VEGF concurrently with a gelatin-PPF composite resulted in similar findings [119]. In this case, a solid PPF rod encapsulated PLGA microparticles either loaded BMP-2 or empty (blank). This rod was encased in a 
crosslinked gelatin cylinder unloaded (blank) or loaded with VEGF. After implantation into rat femoral and subcutaneous defects, the composite constructs were capable of releasing VEGF within the first week of implantation and BMP-2 over at least 50 days (with highest concentrations being released around day 21). By incorporation of a gelation hydrogel into the construct, the two growth factors were released concurrently at different rates.

Ultimately, while delivery of VEGF alone had a significant effect on vessel volume compared to BMP-2 alone and empty scaffolds (although no difference with dual delivery), there was no difference in bone growth between dual delivery and BMP-2 alone groups (both performed significantly better than empty scaffolds and VEGF alone) [119]. GelatinPPF composite constructs can be tailored to release multiple growth factors at different kinetics, allowing for temporal control over drug delivery and resulting in successful bone regeneration in animal models.

\section{Concluding Remarks and Future Directions}

In the past, research surrounding drug delivery from gelatin focused on the interactions between gelatin and loaded drug. The possibility to alter its IEP and crosslinking density has been traditionally investigated as a tool to tune drug release and gelatin degradation kinetics. Nevertheless, gelatin offers high versatility which has resulted in the design of many different drug delivery systems over the last few decades.

Chemical modification of gelatin structure allows for enhanced drug stabilization and higher drug entrapment efficiency of the carrier. The conjugation of specific sequences can also improve the overall biocompatibility of the carrier, as well as result in tissue-specific drug delivery. Given the latest advances in targeted delivery from gelatin-based systems, we envision increased opportunities for gelatin as a delivery vehicle, especially in oncologic applications.

As underscored in this review, gelatin has been successfully incorporated in numerous composite materials, particularly in musculoskeletal tissue engineering. The synergistic use of gelatin with other biomaterials enables higher flexibility in terms of material degradation and controlled release, while maintaining and enhancing the properties of the bulk material (typically a ceramic or other polymer). Gelatin composites have seen success in delivering growth factors within scaffolds to promote tissue regeneration in many in vivo models.

Overall, the modification of gelatin and its combination with other biomaterials demonstrates the flexibility of this biomaterial and ensures its continued role as a vehicle in the fields of drug delivery and tissue engineering.

\section{Acknowledgments}

We acknowledge support by the Army, Navy, NIH, Air Force, VA and Health Affairs to support the AFIRM II effort, under Award No. W81XWH-14-2-0004. The U.S. Army Medical Research Acquisition Activity, 820 Chandler Street, Fort Detrick MD 21702-5014 is the awarding and administering acquisition office. Opinions, interpretations, conclusions and recommendations are those of the author and are not necessarily endorsed by the Department of Defense. We also acknowledge support by the National Institutes of Health (R01 AR048756 and R01 AR057083). AMT acknowledges the Baylor College of Medicine Medical Scientist Training Program (NIH T32 GM007330) and the Barrow Scholars Program. 


\section{References}

1. Ikada Y, Tabata Y. Protein release from gelatin matrices. Adv Drug Deliv Rev. 1998; 31(3):287301. [PubMed: 10837630]

2. Young S, Wong M, Tabata Y, Mikos AG. Gelatin as a delivery vehicle for the controlled release of bioactive molecules. J Control Release. 2005; 109(1-3):256-274. [PubMed: 16266768]

3. Needham CJ, Williams AK, Chew SA, Kasper FK, Mikos AG. Engineering a polymeric gene delivery vector based on poly(ethylenimine) and hyaluronic acid. Biomacromolecules. 2012; 13(5): 1429-1437. [PubMed: 22455481]

4. Pack DW, Hoffman AS, Pun S, Stayton PS. Design and development of polymers for gene delivery. Nat Rev Drug Discov. 2005; 4(7):581-593. [PubMed: 16052241]

5. Hu L, Sun Y, Wu Y. Advances in chitosan-based drug delivery vehicles. Nanoscale. 2013; 5(8): 3103-3111. [PubMed: 23515527]

6. Sanjoh M, Hiki S, Lee Y, Oba M, Miyata K, Ishii T, Kataoka K. Pdna/poly(l-lysine) polyplexes functionalized with a ph-sensitive charge-conversional poly(aspartamide) derivative for controlled gene delivery to human umbilical vein endothelial cells. Macromol Rapid Commun. 2010; 31(13): 1181-1186. [PubMed: 21590873]

7. Nezhadi SH, Choong PF, Lotfipour F, Dass CR. Gelatin-based delivery systems for cancer gene therapy. J Drug Target. 2009; 17(10):731-738. [PubMed: 19863194]

8. Smyth, HDC.; Hickey, AJ. Controlled pulmonary drug delivery. Springer; New York: 2011.

9. Elzoghby AO. Gelatin-based nanoparticles as drug and gene delivery systems: Reviewing three decades of research. J Control Release. 2013; 172(3):1075-1091. [PubMed: 24096021]

10. Olsen D, Yang CL, Bodo M, Chang R, Leigh S, Baez J, Carmichael D, Perala M, Hamalainen ER, Jarvinen M, Polarek J. Recombinant collagen and gelatin for drug delivery. Adv Drug Deliver Rev. 2003; 55(12):1547-1567.

11. Won YW, Kim YH. Recombinant human gelatin nanoparticles as a protein drug carrier. J Control Release. 2008; 127(2):154-161. [PubMed: 18329122]

12. Won YW, Yoon SM, Sonn CH, Lee KM, Kim YH. Nano self-assembly of recombinant human gelatin conjugated with alpha-tocopheryl succinate for hsp90 inhibitor, 17-aag, delivery. Acs Nano. 2011; 5(5):3839-3848. [PubMed: 21517103]

13. Wang H, Boerman OC, Sariibrahimoglu K, Li Y, Jansen JA, Leeuwenburgh SCG. Comparison of micro- vs. Nanostructured colloidal gelatin gels for sustained delivery of osteogenic proteins: Bone morphogenetic protein-2 and alkaline phosphatase. Biomaterials. 2012; 33(33):8695-8703. [PubMed: 22922022]

14. Wang H, Zou Q, Boerman OC, Nijhuis AW, Jansen JA, Li Y, Leeuwenburgh SC. Combined delivery of bmp-2 and bfgf from nanostructured colloidal gelatin gels and its effect on bone regeneration in vivo. J Control Release. 2013; 166(2):172-181. [PubMed: 23266450]

15. Kim K, Lam J, Lu S, Spicer PP, Lueckgen A, Tabata Y, Wong ME, Jansen JA, Mikos AG, Kasper FK. Osteochondral tissue regeneration using a bilayered composite hydrogel with modulating dual growth factor release kinetics in a rabbit model. J Control Release. 2013; 168(2):166-178. [PubMed: 23541928]

16. Kim YH, Furuya H, Tabata Y. Enhancement of bone regeneration by dual release of a macrophage recruitment agent and platelet-rich plasma from gelatin hydrogels. Biomaterials. 2014; 35(1):214224. [PubMed: 24125774]

17. Madan J, Pandey RS, Jain UK, Katare OP, Aneja R, Katyal A. Sterically stabilized gelatin microassemblies of noscapine enhance cytotoxicity, apoptosis and drug delivery in lung cancer cells. Colloids Surf B Biointerfaces. 2013; 107:235-244. [PubMed: 23502046]

18. Gaowa A, Horibe T, Kohno M, Sato K, Harada H, Hiraoka M, Tabata Y, Kawakami K. Combination of hybrid peptide with biodegradable gelatin hydrogel for controlled release and enhancement of anti-tumor activity in vivo. J Control Release. 2014; 176C:1-7. [PubMed: 24378440]

19. Lee SJ, Yhee JY, Kim SH, Kwon IC, Kim K. Biocompatible gelatin nanoparticles for tumortargeted delivery of polymerized sirna in tumor-bearing mice. J Control Release. 2013; 172(1): 358-366. [PubMed: 24036198] 
20. Kimura Y, Tabata Y. Controlled release of stromal-cell-derived factor-1 from gelatin hydrogels enhances angiogenesis. J Biomater Sci Polym Ed. 2010; 21(1):37-51. [PubMed: 20040152]

21. Saito T, Tabata Y. Preparation of gelatin hydrogels incorporating low-molecular-weight heparin for anti-fibrotic therapy. Acta Biomater. 2012; 8(2):646-652. [PubMed: 22079782]

22. Del Gaudio C, Baiguera S, Boieri M, Mazzanti B, Ribatti D, Bianco A, Macchiarini P. Induction of angiogenesis using vegf releasing genipin-crosslinked electrospun gelatin mats. Biomaterials. 2013; 34(31):7754-7765. [PubMed: 23863451]

23. Galaev, I.; Mattiasson, B. Smart polymers : Applications in biotechnology and biomedicine. CRC Press; Boca Raton, FL: 2008.

24. Chen KY, Yao CH. Repair of bone defects with gelatin-based composites: A review. BioMedicine. 2011; 1(1):29-32.

25. Lee EJ, Khan SA, Park JK, Lim KH. Studies on the characteristics of drug-loaded gelatin nanoparticles prepared by nanoprecipitation. Bioprocess Biosyst Eng. 2012; 35(1-2):297-307. [PubMed: 21909678]

26. Rajan M, Raj V. Formation and characterization of chitosan-polylacticacid-polyethylene glycolgelatin nanoparticles: A novel biosystem for controlled drug delivery. Carbohydr Polym. 2013; 98(1):951-958. [PubMed: 23987433]

27. Kumar R, Nagarwal RC, Dhanawat M, Pandit JK. In vitro and in vivo study of indomethacin loaded gelatin nanoparticles. J Biomed Nanotechnol. 2011; 7(3):325-333. [PubMed: 21830472]

28. Narayanan D, Koyakutty GGMLHM, Nair S, Menon D. Poly-(ethylene glycol) modified gelatin nanoparticles for sustained delivery of the anti-inflammatory drug ibuprofen-sodium: An in vitro and in vivo analysis. Nanomedicine. 2013; 9(6):818-828. [PubMed: 23428986]

29. Li WM, Chen SY, Liu DM. In situ doxorubicin-cap shell formation on amphiphilic gelatin-iron oxide core as a multifunctional drug delivery system with improved cytocompatibility, phresponsive drug release and mr imaging. Acta Biomater. 2013; 9(2):5360-5368. [PubMed: 23022876]

30. Wang J, Wu W, Zhang YJ, Wang X, Qian HQ, Liu BR, Jiang XQ. The combined effects of size and surface chemistry on the accumulation of boronic acid-rich protein nanoparticles in tumors. Biomaterials. 2014; 35(2):866-878. [PubMed: 24157313]

31. Bonner DK, Zhao X, Buss H, Langer R, Hammond PT. Crosslinked linear polyethylenimine enhances delivery of DNA to the cytoplasm. J Control Release. 2013; 167(1):101-107. [PubMed: 22995755]

32. Tanigo T, Takaoka R, Tabata Y. Sustained release of water-insoluble simvastatin from biodegradable hydrogel augments bone regeneration. J Control Release. 2010; 143(2):201-206. [PubMed: 20060429]

33. Kommareddy S, Shenoy DB, Amiji MM. Gelatin nanoparticles and their biofunctionalization, Nanotechnologies for the Life Sciences. Biofunctionalization of Nanomaterials. 2005; 1:330-352.

34. Obata Y, Nishino T, Kushibiki T, Tomoshige R, Xia Z, Miyazaki M, Abe K, Koji T, Tabata Y, Kohno S. Hsp47 sirna conjugated with cationized gelatin microspheres suppresses peritoneal fibrosis in mice. Acta Biomater. 2012; 8(7):2688-2696. [PubMed: 22487929]

35. Tseng CL, Su WY, Yen KC, Yang KC, Lin FH. The use of biotinylated-egf-modified gelatin nanoparticle carrier to enhance cisplatin accumulation in cancerous lungs via inhalation. Biomaterials. 2009; 30(20):3476-3485. [PubMed: 19345990]

36. Lee GY, Park K, Nam JH, Kim SY, Byun Y. Anti-tumor and anti-metastatic effects of gelatindoxorubicin and pegylated gelatin-doxorubicin nanoparticles in scc7 bearing mice. J Drug Target. 2006; 14(10):707-716. [PubMed: 17162740]

37. Kommareddy S, Amiji M. Poly(ethylene glycol)-modified thiolated gelatin nanoparticles for glutathione-responsive intracellular DNA delivery. Nanomedicine. 2007; 3(1):32-42. [PubMed: 17379167]

38. Ishikawa H, Nakamura Y, Jo J, Tabata Y. Gelatin nanospheres incorporating sirna for controlled intracellular release. Biomaterials. 2012; 33(35):9097-9104. [PubMed: 22985993]

39. Li WM, Liu DM, Chen SY. Amphiphilically-modified gelatin nanoparticles: Self-assembly behavior, controlled biodegradability, and rapid cellular uptake for intracellular drug delivery. J Mater Chem. 2011; 21(33):12381-12388. 
40. Magadala P, Amiji M. Epidermal growth factor receptor-targeted gelatin-based engineered nanocarriers for DNA delivery and transfection in human pancreatic cancer cells. AAPS J. 2008; 10(4):565-576. [PubMed: 19034673]

41. Tseng CL, Wu SY, Wang WH, Peng CL, Lin FH, Lin CC, Young TH, Shieh MJ. Targeting efficiency and biodistribution of biotinylated-egf-conjugated gelatin nanoparticles administered via aerosol delivery in nude mice with lung cancer. Biomaterials. 2008; 29(20):3014-3022. [PubMed: 18436301]

42. Tian XH, Wei F, Wang TX, Wang D, Wang J, Lin XN, Wang P, Ren L. Blood-brain barrier transport of tat peptide and polyethylene glycol decorated gelatin-siloxane nanoparticle. Materials Letters. 2012; 68:94-96.

43. Knop K, Hoogenboom R, Fischer D, Schubert US. Poly(ethylene glycol) in drug delivery: Pros and cons as well as potential alternatives. Angew Chem Int Edit. 2010; 49(36):6288-6308.

44. Jevsevar S, Kunstelj M, Porekar VG. Pegylation of therapeutic proteins. Biotechnol J. 2010; 5(1): 113-128. [PubMed: 20069580]

45. Kaul G, Amiji M. Long-circulating poly(ethylene glycol)-modified gelatin nanoparticles for intracellular delivery. Pharm Res-Dordr. 2002; 19(7):1061-1067.

46. Einerson NJ, Stevens KR, Kao WYJ. Synthesis and physicochemical analysis of gelatin-based hydrogels for drug carrier matrices. Biomaterials. 2003; 24(3):509-523. [PubMed: 12423606]

47. Lammers T, Kiessling F, Hennink WE, Storm G. Drug targeting to tumors: Principles, pitfalls and (pre-) clinical progress. J Control Release. 2012; 161(2):175-187. [PubMed: 21945285]

48. Danhier F, Feron O, Preat V. To exploit the tumor microenvironment: Passive and active tumor targeting of nanocarriers for anti-cancer drug delivery. J Control Release. 2010; 148(2):135-146. [PubMed: 20797419]

49. Uesugi Y, Kawata H, Jo J, Saito Y, Tabata Y. An ultrasound-responsive nano delivery system of tissue-type plasminogen activator for thrombolytic therapy. J Control Release. 2010; 147(2):269277. [PubMed: 20696194]

50. Mimi H, Ho KM, Siu YS, Wu A, Li P. Polyethyleneimine-based core-shell nanogels: A promising sirna carrier for argininosuccinate synthetase mrna knockdown in hela cells. J Control Release. 2012; 158(1):123-130. [PubMed: 22094103]

51. Kushibiki T, Matsuoka H, Tabata Y. Synthesis and physical characterization of poly(ethylene glycol)-gelatin conjugates. Biomacromolecules. 2004; 5(1):202-208. [PubMed: 14715027]

52. Doi N, Jo JI, Tabata Y. Preparation of biodegradable gelatin nanospheres with a narrow size distribution for carrier of cellular internalization of plasmid DNA. J Biomat Sci-Polym E. 2012; 23(8):991-1004.

53. Kuo WT, Huang HY, Chou MJ, Wu MC, Huang YY. Surface modification of gelatin nanoparticles with polyethylenimine as gene vector. J Nanomater. 2011

54. Zorzi GK, Contreras-Ruiz L, Parraga JE, Lopez-Garcia A, Bello RR, Diebold Y, Seijo B, Sanchez A. Expression of muc5ac in ocular surface epithelial cells using cationized gelatin nanoparticles. Mol Pharmaceut. 2011; 8(5):1783-1788.

55. Zorzi GK, Párraga JE, Seijo B, Sánchez A. Hybrid nanoparticle design based on cationized gelatin and the polyanions dextran sulfate and chondroitin sulfate for ocular gene therapy. Macromol Biosci. 2011; 11(7):905-913. [PubMed: 21480529]

56. Wu DC, Wan MX. A novel fluoride anion modified gelatin nanogel system for ultrasoundtriggered drug release. J Pharm Pharm Sci. 2008; 11(4):32-45. [PubMed: 19183512]

57. Han SY, Li MJ, Liu XG, Gao HX, Wu Y. Construction of amphiphilic copolymer nanoparticles based on gelatin as drug carriers for doxorubicin delivery. Colloid Surface B. 2013; 102:833-841.

58. Tran PHL, Tran TTD, Lee BJ. Enhanced solubility and modified release of poorly water-soluble drugs via self-assembled gelatin-oleic acid nanoparticles. Int J Pharm. 2013; 455(1-2):235-240. [PubMed: 23876500]

59. Park K. Biomaterials for cancer therapeutics : Diagnosis, prevention and therapy. 2013

60. Wheeler DL, Dunn EF, Harari PM. Understanding resistance to egfr inhibitors-impact on future treatment strategies. Nat Rev Clin Oncol. 2010; 7(9):493-507. [PubMed: 20551942]

61. Wei B, Wei Y, Zhang K, Wang J, Xu R, Zhan S, Lin G, Wang W, Liu M, Wang L, Zhang R, Li J. Development of an antisense rna delivery system using conjugates of the ms 2 bacteriophage 
capsids and hiv-1 tat cell-penetrating peptide. Biomed Pharmacother. 2009; 63(4):313-318. [PubMed: 18823738]

62. Habraken WJ, de Jonge LT, Wolke JG, Yubao L, Mikos AG, Jansen JA. Introduction of gelatin microspheres into an injectable calcium phosphate cement. J Biomed Mater Res A. 2008; 87(3): 643-655. [PubMed: 18189298]

63. Habraken WJ, Boerman OC, Wolke JG, Mikos AG, Jansen JA. In vitro growth factor release from injectable calcium phosphate cements containing gelatin microspheres. J Biomed Mater Res A. 2009; 91(2):614-622. [PubMed: 18985784]

64. Yao KD, Yin YJ, Xu MX, Wang YF. Investigation of ph-sensitive drug-delivery system of chitosan/gelatin hybrid polymer network. Polymer international. 1995; 38(1):77-82.

65. Tan H, Huang D, Lao L, Gao C. Rgd modified plga/gelatin microspheres as microcarriers for chondrocyte delivery. J Biomed Mater Res B Appl Biomater. 2009; 91(1):228-238. [PubMed: 19388090]

66. Motsitsi NS. Management of infected nonunion of long bones: The last decade (1996-2006). Injury. 2008; 39(2):155-160. [PubMed: 18234202]

67. Dressler M, Dombrowski F, Simon U, Börnstein J, Hodoroaba V, Feigl M, Grunow S, Gildenhaar $\mathrm{R}$, Neumann M. Influence of gelatin coatings on compressive strength of porous hydroxyapatite ceramics. Journal of the European Ceramic Society. 2011; 31(4):523-529.

68. Kim HW, Knowles JC, Kim HE. Porous scaffolds of gelatin-hydroxyapatite nanocomposites obtained by biomimetic approach: Characterization and antibiotic drug release. J Biomed Mater Res B Appl Biomater. 2005; 74(2):686-698. [PubMed: 15988752]

69. Teng S, Chen L, Guo Y, Shi J. Formation of nano-hydroxyapatite in gelatin droplets and the resulting porous composite microspheres. J Inorg Biochem. 2007; 101(4):686-691. [PubMed: 17316810]

70. Sivakumar M, Panduranga Rao K. Preparation, characterization and in vitro release of gentamicin from coralline hydroxyapatite-gelatin composite microspheres. Biomaterials. 2002; 23(15):31753181. [PubMed: 12102189]

71. Perez RA, Del Valle S, Altankov G, Ginebra MP. Porous hydroxyapatite and gelatin/ hydroxyapatite microspheres obtained by calcium phosphate cement emulsion. J Biomed Mater Res B Appl Biomater. 2011; 97(1):156-166. [PubMed: 21290594]

72. Ginebra MP, Traykova T, Planell JA. Calcium phosphate cements as bone drug delivery systems: A review. J Control Release. 2006; 113(2):102-110. [PubMed: 16740332]

73. Li M, Liu X, Ge B. Calcium phosphate cement with bmp-2-loaded gelatin microspheres enhances bone healing in osteoporosis: A pilot study. Clin Orthop Relat Res. 2010; 468(7):1978-1985. [PubMed: 20306162]

74. Liao H, Walboomers XF, Habraken WJ, Zhang Z, Li Y, Grijpma DW, Mikos AG, Wolke JG, Jansen JA. Injectable calcium phosphate cement with plga, gelatin and ptmc microspheres in a rabbit femoral defect. Acta Biomater. 2011; 7(4):1752-1759. [PubMed: 21185953]

75. van der Rest M, Garrone R. Collagen family of proteins. FASEB J. 1991; 5(13):2814-2823. [PubMed: 1916105]

76. Wan ACA, Tai BCU. Chitin - a promising biomaterial for tissue engineering and stem cell technologies. Biotechnol Adv. 2013; 31(8):1776-1785. [PubMed: 24080076]

77. Kundu B, Kurland NE, Bano S, Patra C, Engel FB, Yadavalli VK, Kundu SC. Silk proteins for biomedical applications: Bioengineering perspectives. Progress in Polymer Science. 2014; 39(2): 251-267.

78. Allison DD, Grande-Allen KJ. Review. Hyaluronan: A powerful tissue engineering tool. Tissue Eng. 2006; 12(8):2131-2140. [PubMed: 16968154]

79. Toole BP. Hyaluronan and its binding proteins, the hyaladherins. Current Opinion in Cell Biology. 1990; 2(5):839-844. [PubMed: 1707285]

80. Luo Y, Kirker KR, Prestwich GD. Cross-linked hyaluronic acid hydrogel films: New biomaterials for drug delivery. J Control Release. 2000; 69(1):169-184. [PubMed: 11018555]

81. Shu XZ, Liu YC, Palumbo F, Prestwich GD. Disulfide-crosslinked hyaluronan-gelatin hydrogel films: A covalent mimic of the extracellular matrix for in vitro cell growth. Biomaterials. 2003; 24(21):3825-3834. [PubMed: 12818555] 
82. Peattie RA, Pike DB, Yu B, Cai S, Shu XZ, Prestwich GD, Firpo MA, Fisher RJ. Effect of gelatin on heparin regulation of cytokine release from hyaluronan-based hydrogels. Drug Deliv. 2008; 15(6):389-397. [PubMed: 18686083]

83. Hosack LW, Firpo MA, Scott JA, Prestwich GD, Peattie RA. Microvascular maturity elicited in tissue treated with cytokine-loaded hyaluronan-based hydrogels. Biomaterials. 2008; 29(15):23362347. [PubMed: 18313745]

84. Li J, He A, Zheng J, Han CC. Gelatin and gelatin-hyaluronic acid nanofibrous membranes produced by electrospinning of their aqueous solutions. Biomacromolecules. 2006; 7(7):22432247. [PubMed: 16827594]

85. Sinha VR, Singla AK, Wadhawan S, Kaushik R, Kumria R, Bansal K, Dhawan S. Chitosan microspheres as a potential carrier for drugs. Int J Pharm. 2004; 274(1-2):1-33. [PubMed: 15072779]

86. Thacharodi D, Panduranga Rao K. Collagen-chitosan composite membranes for controlled release of propranolol hydrochloride. Int J Pharm. 1995; 120(1):115-118.

87. Zhao F, Yin Y, Lu WW, Leong JC, Zhang W, Zhang J, Zhang M, Yao K. Preparation and histological evaluation of biomimetic three-dimensional hydroxyapatite/chitosan-gelatin network composite scaffolds. Biomaterials. 2002; 23(15):3227-3234. [PubMed: 12102194]

88. Zhao F, Grayson WL, Ma T, Bunnell B, Lu WW. Effects of hydroxyapatite in 3-d chitosan-gelatin polymer network on human mesenchymal stem cell construct development. Biomaterials. 2006; 27(9):1859-1867. [PubMed: 16225916]

89. Liu H, Fan H, Cui Y, Chen Y, Yao K, Goh JC. Effects of the controlled-released basic fibroblast growth factor from chitosan-gelatin microspheres on human fibroblasts cultured on a chitosangelatin scaffold. Biomacromolecules. 2007; 8(5):1446-1455. [PubMed: 17375950]

90. Wang Y, Kim HJ, Vunjak-Novakovic G, Kaplan DL. Stem cell-based tissue engineering with silk biomaterials. Biomaterials. 2006; 27(36):6064-6082. [PubMed: 16890988]

91. Shubhra QT, Alam A, Beg M. Mechanical and degradation characteristics of natural silk fiber reinforced gelatin composites. Materials Letters. 2011; 65(2):333-336.

92. Mandal BB, Mann JK, Kundu SC. Silk fibroin/gelatin multilayered films as a model system for controlled drug release. Eur J Pharm Sci. 2009; 37(2):160-171. [PubMed: 19429423]

93. Gil ES, Spontak RJ, Hudson SM. Effect of beta-sheet crystals on the thermal and rheological behavior of protein-based hydrogels derived from gelatin and silk fibroin. Macromol Biosci. 2005; 5(8):702-709. [PubMed: 16080165]

94. Gil ES, Frankowski DJ, Spontak RJ, Hudson SM. Swelling behavior and morphological evolution of mixed gelatin/silk fibroin hydrogels. Biomacromolecules. 2005; 6(6):3079-3087. [PubMed: 16283730]

95. Lutolf MP, Hubbell JA. Synthetic biomaterials as instructive extracellular microenvironments for morphogenesis in tissue engineering. Nat Biotechnol. 2005; 23(1):47-55. [PubMed: 15637621]

96. Anderson JM, Rodriguez A, Chang DT. Foreign body reaction to biomaterials. Semin Immunol. 2008; 20(2):86-100. [PubMed: 18162407]

97. Persson GR, Salvi GE, Heitz-Mayfield LJ, Lang NP. Antimicrobial therapy using a local drug delivery system (arestin) in the treatment of peri-implantitis. I: Microbiological outcomes. Clin Oral Implants Res. 2006; 17(4):386-393. [PubMed: 16907769]

98. Tracy MA, Ward KL, Firouzabadian L, Wang Y, Dong N, Qian R, Zhang Y. Factors affecting the degradation rate of poly(lactide-co-glycolide) microspheres in vivo and in vitro. Biomaterials. 1999; 20(11):1057-1062. [PubMed: 10378806]

99. Zeng J, Xu X, Chen X, Liang Q, Bian X, Yang L, Jing X. Biodegradable electrospun fibers for drug delivery. J Control Release. 2003; 92(3):227-231. [PubMed: 14568403]

100. Meng ZX, Xu XX, Zheng W, Zhou HM, Li L, Zheng YF, Lou X. Preparation and characterization of electrospun plga/gelatin nanofibers as a potential drug delivery system. Colloids Surf B Biointerfaces. 2011; 84(1):97-102. [PubMed: 21227661]

101. Meng Z, Wang Y, Ma C, Zheng W, Li L, Zheng Y. Electrospinning of plga/gelatin randomlyoriented and aligned nanofibers as potential scaffold in tissue engineering. Materials Science and Engineering: C. 2010; 30(8):1204-1210. 
102. Holland TA, Tabata Y, Mikos AG. In vitro release of transforming growth factor-beta 1 from gelatin microparticles encapsulated in biodegradable, injectable oligo(poly(ethylene glycol) fumarate) hydrogels. J Control Release. 2003; 91(3):299-313. [PubMed: 12932709]

103. Lam J, Kim K, Lu S, Tabata Y, Scott DW, Mikos AG, Kurtis Kasper F. A factorial analysis of the combined effects of hydrogel fabrication parameters on the in vitro swelling and degradation of oligo(poly(ethylene glycol) fumarate) hydrogels. J Biomed Mater Res A. 2013:n/a-n/a.

104. Temenoff JS, Park H, Jabbari E, Conway DE, Sheffield TL, Ambrose CG, Mikos AG. Thermally cross-linked oligo(poly(ethylene glycol) fumarate) hydrogels support osteogenic differentiation of encapsulated marrow stromal cells in vitro. Biomacromolecules. 2004; 5(1):5-10. [PubMed: 14715001]

105. Holland TA, Bodde EW, Baggett LS, Tabata Y, Mikos AG, Jansen JA. Osteochondral repair in the rabbit model utilizing bilayered, degradable oligo(poly(ethylene glycol) fumarate) hydrogel scaffolds. J Biomed Mater Res A. 2005; 75(1):156-167. [PubMed: 16052490]

106. Kasper FK, Kushibiki T, Kimura Y, Mikos AG, Tabata Y. In vivo release of plasmid DNA from composites of oligo(poly(ethylene glycol)fumarate) and cationized gelatin microspheres. J Control Release. 2005; 107(3):547-561. [PubMed: 16139915]

107. Nejadnik MR, Mikos AG, Jansen JA, Leeuwenburgh SC. Facilitating the mineralization of oligo(poly(ethylene glycol) fumarate) hydrogel by incorporation of hydroxyapatite nanoparticles. J Biomed Mater Res A. 2012; 100(5):1316-1323. [PubMed: 22374694]

108. Holland TA, Tabata Y, Mikos AG. Dual growth factor delivery from degradable oligo(poly(ethylene glycol) fumarate) hydrogel scaffolds for cartilage tissue engineering. $\mathrm{J}$ Control Release. 2005; 101(1-3):111-125. [PubMed: 15588898]

109. Holland TA, Tessmar JK, Tabata Y, Mikos AG. Transforming growth factor-beta 1 release from oligo(poly(ethylene glycol) fumarate) hydrogels in conditions that model the cartilage wound healing environment. J Control Release. 2004; 94(1):101-114. [PubMed: 14684275]

110. Suggs, LJ.; Moore, SA.; Mikos, AG. Physical properties of polymers handbook. Springer; 2007. p. 939-950.

111. Fisher JP, Lalani Z, Bossano CM, Brey EM, Demian N, Johnston CM, Dean D, Jansen JA, Wong ME, Mikos AG. Effect of biomaterial properties on bone healing in a rabbit tooth extraction socket model. J Biomed Mater Res A. 2004; 68(3):428-438. [PubMed: 14762922]

112. Lee KW, Wang S, Fox BC, Ritman EL, Yaszemski MJ, Lu L. Poly(propylene fumarate) bone tissue engineering scaffold fabrication using stereolithography: Effects of resin formulations and laser parameters. Biomacromolecules. 2007; 8(4):1077-1084. [PubMed: 17326677]

113. Fisher JP, Vehof JW, Dean D, van der Waerden JP, Holland TA, Mikos AG, Jansen JA. Soft and hard tissue response to photocrosslinked poly(propylene fumarate) scaffolds in a rabbit model. J Biomed Mater Res. 2002; 59(3):547-556. [PubMed: 11774313]

114. Yaszemski MJ, Payne RG, Hayes WC, Langer RS, Aufdemorte TB, Mikos AG. The ingrowth of new bone tissue and initial mechanical properties of a degrading polymeric composite scaffold. Tissue Eng. 1995; 1(1):41-52. [PubMed: 19877914]

115. Dean D, Topham NS, Meneghetti SC, Wolfe MS, Jepsen K, He S, Chen JE, Fisher JP, Cooke M, Rimnac C, Mikos AG. Poly(propylene fumarate) and poly(dl-lactic-co-glycolic acid) as scaffold materials for solid and foam-coated composite tissue-engineered constructs for cranial reconstruction. Tissue Eng. 2003; 9(3):495-504. [PubMed: 12857417]

116. Lewandrowski KU, Cattaneo MV, Gresser JD, Wise DL, White RL, Bonassar L, Trantolo DJ. Effect of a poly(propylene fumarate) foaming cement on the healing of bone defects. Tissue Eng. 1999; 5(4):305-316. [PubMed: 10477853]

117. Patel ZS, Ueda H, Yamamoto M, Tabata Y, Mikos AG. In vitro and in vivo release of vascular endothelial growth factor from gelatin microparticles and biodegradable composite scaffolds. Pharm Res. 2008; 25(10):2370-2378. [PubMed: 18663411]

118. Patel ZS, Young S, Tabata Y, Jansen JA, Wong ME, Mikos AG. Dual delivery of an angiogenic and an osteogenic growth factor for bone regeneration in a critical size defect model. Bone. 2008; 43(5):931-940. [PubMed: 18675385] 
119. Kempen DH, Lu L, Heijink A, Hefferan TE, Creemers LB, Maran A, Yaszemski MJ, Dhert WJ. Effect of local sequential vegf and bmp-2 delivery on ectopic and orthotopic bone regeneration. Biomaterials. 2009; 30(14):2816-2825. [PubMed: 19232714] 


\section{Table 1}

\section{Examples of Gelatin Modifications}

\begin{tabular}{|c|c|c|}
\hline Modification outcomes & Advantages & Applications \\
\hline Stealth delivery & $\begin{array}{ll}\text { - } & \text { Reduced immunogenicity } \\
\text { - } & \text { Prolonged circulatory time } \\
\text { - } & \begin{array}{l}\text { Evasion of reticuloendothelial } \\
\text { uptake }\end{array}\end{array}$ & $\begin{array}{l}\text { PEGylated nanoparticles for pulmonary drug } \\
\text { delivery }[17,36] \\
\text { - } \\
\text { PEGylated/thiolated nanoparticles for } \\
\text { intracellular DNA delivery [37] } \\
\text { - } \quad \begin{array}{l}\text { PEGylated nanocarriers for anti-inflammatory } \\
\text { drug release [28] }\end{array}\end{array}$ \\
\hline $\begin{array}{l}\text { Enhanced drug stabilization } \\
\text { and loading efficiency }\end{array}$ & $\begin{array}{l}\text { - Tunable hydrophobicity of the } \\
\text { carrier } \\
\text { - } \quad \text { Higher drug encapsulation } \\
\text { efficiency } \\
\text { - } \quad \begin{array}{l}\text { More stable complexation between } \\
\text { carrier and drug }\end{array}\end{array}$ & $\begin{array}{l}\text { - } \quad \text { Succinylated gelatin for angiogenesis [20] } \\
\text { - } \quad \text { Cationized microparticles for siRNA delivery } \\
{[34,38]} \\
\text { - } \quad \begin{array}{l}\text { Hexanoyl anhydride grafted-nanoparticles for } \\
\text { anticancer drug release [39] }\end{array}\end{array}$ \\
\hline Targeted drug delivery & $\begin{array}{ll}\text { - } & \text { Highly selective cell targeting } \\
\text { - } & \text { Reduced side-effects } \\
\text { - } & \text { Lower systemic cytotoxicity }\end{array}$ & $\begin{array}{l}\text { - Introduction of EGFR-recognition sequence for } \\
\text { gene delivery in pancreatic cancer [40] } \\
\text { - } \quad \text { EGF-modified nanoparticles for aerosol delivery } \\
\text { to lung cancer [35, 41] } \\
\text { - } \quad \begin{array}{l}\text { PEG/peptide conjugated nanoparticles for brain } \\
\text { drug delivery [42] }\end{array}\end{array}$ \\
\hline
\end{tabular}




\section{Table 2}

\section{Examples of Gelatin Composite Systems}

\begin{tabular}{|c|c|c|c|}
\hline Composite material class & Complementary material strength & $\begin{array}{l}\text { Material weakness addressed } \\
\text { with gelatin }\end{array}$ & Applications \\
\hline Ceramics & $\begin{array}{ll}\text { - } & \text { High compressive properties } \\
\text { - } & \text { Osteoconductive }\end{array}$ & - $\quad$ Slow biodegradability & $\begin{array}{l}\text { Calcium } \\
\text { phosphate } \\
\text { cement with } \\
\text { embedded } \\
\text { gelatin } \\
\text { microparticles } \\
{[62,63]}\end{array}$ \\
\hline Naturally-derived polymers & $\begin{array}{l}\text { - } \quad \text { Biomimetic } \\
\text { - } \quad \text { Protein or polysaccharide-based }\end{array}$ & - $\quad$ Lack of IEP tunability & $\begin{array}{l}\text { Gelatin- } \\
\text { chitosan } \\
\text { sponges for } \\
\text { wound } \\
\text { treatment [64] }\end{array}$ \\
\hline Synthetic polymers & - Highly-tunable properties & $\begin{array}{l}\text { - No inherent cell- } \\
\text { recognition sites }\end{array}$ & $\begin{array}{ll}\text { - Gelatin- } \\
\text { poly(lactic-co- } \\
\text { glycolic acid) } \\
\text { microparticles } \\
\text { loaded with } \\
\text { chondrocytes } \\
\text { [65] }\end{array}$ \\
\hline
\end{tabular}

